\title{
Results of pars plana vitrectomy for primary rhegmatogenous retinal detachment with PVR grades $A$ and $B$ in high-myopic eyes
}

\author{
Ladislav Janco $($ ) $\cdot$ Katarina Tkacova-Villemova $\cdot$ Anna Matisovska • \\ Maria Mesarosova - Marta Ondrejkova - Alexandra Kollarova • \\ Matus Rehak
}

Received: 11 November 2018/Accepted: 22 May 2019/Published online: 25 May 2019

(C) The Author(s) 2019

\begin{abstract}
Purpose To evaluate the results of pars plana vitrectomy (PPV) without adjuvant buckling procedures for the primary rhegmatogenous retinal detachment (RRD) with PVR grades A and B in high-myopic eyes.

Methods A retrospective review included 291 eyes treated for primary RRD from 2008 to 2016. The single surgery success rate (SSSR), the total number of surgeries, outcomes and complications were analysed between group of 67 eyes with high axial myopia (group A) and group of 224 eyes without high myopia (group B).

Results The mean follow-up was $30.6 \pm 22.8$ months. The SSSR in group A was $82.1 \%$ and in group B was $86.2 \%(p>0.05)$. The final reattachment rate and number of required surgeries were in group A $96.3 \%$ (3.1 surgeries) and in group B 96.0\% (2.8 surgeries). The initial BCVA improved in group A from 1.58 to 0.58 LogMAR at year 3; and in group B from 1.21 to 0.34
\end{abstract}

L. Janco $(\bowtie) \cdot$ K. Tkacova-Villemova .

A. Matisovska - M. Mesarosova - M. Ondrejkova .

A. Kollarova $\cdot$ M. Rehak

2nd Eye Clinic of Slovak Medical University,

F. D. Roosevelt Hospital, Nám. L. Svobodu 1,

97401 Banská Bystrica, Slovakia

e-mail: ljanco@nspbb.sk;

jancovci@zoznam.sk

M. Rehak

Department of Ophthalmology, University of Leipzig,

Leipzig, Germany
LogMAR. In match-pair analysis of macula-off RRD, no significant difference of the CRT between groups A and $\mathrm{B}$ was observed within 3 years of follow-up.

Conclusion The anatomical success of primary PPV for RRD did not differ between high-myopic and nonhigh-myopic eyes in PVR grades A and B. However, functional results of high-myopic eyes are worse compared to eyes without high axial myopia.

Keywords Retinal detachment $\cdot$ Myopia $\cdot$ Pars plana vitrectomy · OCT

\section{Introduction}

Myopia is a common ocular condition, and it is not just a simple refractive error; however, it is an eyesightthreatening disease. In the year 2000, the prevalence of high myopia was about $2.7 \%$ of the world population (163 million people) and it is predicted that by 2050 there will be about 938 million people suffering from high myopia (9.8\% of the world population) [1]. High myopia may result in irreversible visual loss due to the retinal detachment, choroidal neovascularization, macular atrophy, glaucoma, cataract and other complications [1-4].

Although the anatomical success rate of the vitreoretinal surgeries, especially pars plana vitrectomy (PPV), has improved over the past years, the rhegmatogenous retinal detachment (RRD) surgery of 
high-myopic eyes is still challenging for the vitreoretinal surgeons. Shifting of the primary surgical approach for RD towards PPV has been also observed [5]. The surgery of high-myopic eyes differs from nonhigh-myopic eyes due to the following facts: the very long axial length, the fragile and thin sclera, the choroidal hemodynamic changes with an increased risk of the subretinal and choroid haemorrhage, the choroidal detachment, the anomalous posterior vitreous detachment with the high prevalence of vitreoschisis, the pathologic vitreoretinal attachments with higher retinal break occurrence, as well as the irregularity of the posterior border of the vitreous base. In comparison with the general population, the anatomical single surgery success rate (SSSR) of retinal detachment surgery in high-myopic eyes was accepted to be lower [6].

The purpose of our study was to compare the longterm anatomical and functional results of 67 eyes with high myopia with 224 non-high-myopic eyes treated with PPV without adjuvant buckling procedures for the primary RRD in our clinic.

\section{Materials and methods}

We retrospectively reviewed the charts of 336 consecutive eyes with primary RRD which underwent surgery at the 2nd Eye Clinic of Slovak Medical University, F. D. Roosevelt Hospital, Banská Bystrica, Slovakia, from January 2008 to July 2016.

Eyes were included into the analysis if the following criteria were met: (1) primary RRD with PVR A and $\mathrm{B}$ with pre- or intraoperatively detected retinal break was treated with 20 or 23 gauge PPV without adjuvant buckling surgery; (2) duration of RRD less than 2 months; (3) follow-up time $\geq 6$ months after primary surgery.

Exclusion criteria for the evaluation were: (1) presence of PVR grade C; (2) any previous intraocular surgical intervention except cataract surgery $\geq 3$ months prior to RRD; (3) previous unsuccessful buckling surgery for RRD; (4) terminal glaucoma eye damage; (5) heredodegenerative retinal diseases and/or exsudative age-related macular degeneration (AMD).

The retrospective data analysis was approved by the Institutional Review Board of F. D. Roosevelt Hospital in Banská Bystrica, and it was adherent to the
Declaration of Helsinki. All patients gave their informed consent prior to their treatment.

According to the axial length (AXL) measured by ultrasound biometry (Compact Touch, Quantel medical, France), patients were divided into two subgroups. Group A (high-myopic eyes) included patients with $\mathrm{AXL} \geq 26.0 \mathrm{~mm}$. Patients with $\mathrm{AXL}<26.0$ $\mathrm{mm}$ were included in group $\mathrm{B}$ (non-high-myopic eyes).

At baseline and during the follow-up time (months $3,6,9,12$ and years 2, 3, 5), the following ophthalmologic examinations were performed: BCVA, slit-lamp biomicroscopy, fundus examination and postoperative measurements of the central retina thickness CRT using Spectralis OCT (Heidelberg Engineering, Heidelberg, Germany). BCVA values measured in decimal visual acuity were converted into LogMAR using the Freiburger visual acuity conversion chart [7].

The single surgery success rate (SSSR), the total number of required surgeries, visual and morphological outcomes and complications were analysed between both subgroups of patients. In silicone oilfilled eyes, silicone oil removal was performed in 3-6 months after primary surgery. SSSR was defined as reattached retina at least 6 months after silicone oil removal.

Retinal detachment repair

In all eyes, total vitrectomy was performed using the 20 or 23 gauge (Constellation vision system, Alcon1 Laboratories, Inc.) under vitreous visualization with triamcinolone acetonide $(40 \mathrm{mg} / \mathrm{mL}$ diluted with balanced salt solution) and then was followed by internal subretinal fluid drainage through fluid-air exchange and laser photocoagulation or cryoretinopexy applied around retinal tears. Fluid-gas exchange (air, SF6, C3F8) or fluid-silicon oil exchange was performed in all eyes. The use of silicone oil as primary vitreous tamponade we always consider very carefully. We have considered the general health status, positioning ability, the need to travel and the lens status (e.g. aphakic eyes).

In eyes of RRD with epiretinal membrane, the epiretinal membrane removal and primary peeling of internal limiting membrane after staining with Brilliant Blue G $\left(0.025 \%\right.$, ILM-BLUE ${ }^{\circledR}$; DORC) were performed. If there was clinically significant lens 
opacity preoperatively, phacoemulsification and intraocular lens implantation were performed.

\section{Statistical analysis}

The qualitative variables are demonstrated using the absolute and relative multiplicity. The difference referring to a qualitative variable was tested by Fisher's exact test (at the contingency table $2 \times 2$ ) or Chi-Quadrate Test of Independence (the contingency table of bigger scale than $2 \times 2$ ). The testing using the Chi-Quadrate Test of Independence was performed when the following condition was met: the share of anticipated multiplicities with the value less than 5 is less than $20 \%$. In other cases, the testing was not executed. $T$ test or the scatter analysis (ANOVA) tests the difference referring to a qualitative variable according to the number of the compared groups. All used tests are reciprocal. The value $\alpha=0.05$ was chosen to be the significance level.

\section{Results}

From a total of 336 screened eyes 291 eyes of 281 patients (137 men (48.8\%) and 144 women $(51.2 \%)$ ) were included into the analysis. Sixty-seven eyes (23\%) met the criteria of high myopia and were included in the subgroup A; subgroup B included in total 224 (77\%) non-high-myopic eyes. The baseline parameters are presented in Table 1.

Mean age of all patients at the baseline (at time of surgery) was $60.2 \pm 12.5$ years (range 18-86 years). Most of the patients underwent the primary surgery of RD between 50 and 69 years of age $(65.3 \%), 13.7 \%$ of patients were younger than 50 , and $21.0 \%$ of patients were older than 70 . The mean age of women was $61.8 \pm 11.8$ years, and in men $58.5 \pm 13.0$ years. The age at baseline differs significantly between groups A and B. As displayed in Table 1, patients with high myopia experienced their first RRD about 10 years earlier than patients without myopia $(53.8 \pm 14.7$ vs. $62.1 \pm 11.1$ years in group A vs group B, respectively). The mean follow-up time was $30.6 \pm 22.8$ months (range 6-96 months). The data from follow-up visits at years 1, 2, 3 and 4 were available from $244(83.8 \%)$, $162(55.7 \%), 102(35.1 \%)$ and $50(17.2 \%)$ eyes, respectively. The follow-up of 7 years achieved 12 eyes $(4.1 \%)$ only. Due to the small number of highmyopic eyes (group A) with follow-up at years 5 and 7 (16 and 3 eyes), no statistical analysis for these time points was possible. We have recorded myopia in 144 eyes (49.5\%), out of which the high myopia in 67 eyes (23\%) was found. The duration of RD at day of first surgery was $<7$ days in 25 eyes (37.3\%) in group A and 97 eyes (43.3\%) in group B. \%). In group A, the presence of macula-off RRD at baseline was significantly higher than in group B [60 of 67 eyes $(89.6 \%)$ versus 166 of $224(74.1 \%)$ eyes; $p=0.008]$. The 20-gauge pars plana vitrectomy was performed in 130 eyes (44.7\%), and 23-gauge PPV was preformed in 161 eyes $(55.3 \%)$. Primary tamponade with air was used in 4 eyes $(1.4 \%)$, SF6 in 72 eyes $(24.7 \%), \mathrm{C} 3 \mathrm{~F} 8$ in 170 eyes $(58.4 \%)$ and silicone oil in 45 eyes $(15.5 \%)$. There were no significant differences between groups $\mathrm{A}$ and $\mathrm{B}$ in the presence of the peripheral retinal degenerations, number and localization of the retinal breaks and holes and number of RRD quadrants (Table 1). The phacoemulsification for the cataract progression after PPV was performed in 35 of 184 primary phakic eyes (19.0\%) within 6 months after PPV, in another 86 eyes $(46.7 \%)$ within 1 year and in 35 eyes $(19.0 \%)$ within 3 years. In total, 28 eyes $(15.2 \%)$ had clear lens until the end of follow-up.

\section{Anatomical results}

The anatomical SSSR (displayed in Fig. 1) did not differ between both evaluated subgroups and was $82.1 \%$, and $86.2 \%$ in eyes with high myopia and nonhigh-myopic eyes, respectively $(p>0.05)$. Within 3 years after the initial surgery in total, 3.1 surgeries were required in group A and 2.8 in group B to achieve the anatomic success rates of $96.3 \%$ and $96.0 \%$, respectively (see Fig. 1).

The incidence of development of postoperative epiretinal membrane up to the last follow-up visit did not significantly differ $(p=0.541)$ between the group A in 7 eyes (10.4\%) and group B in 32 eyes (14.3\%).

Vitreous tamponade

The used primary vitreous tamponade is shown in Fig. 2. The most frequently used tamponade in both evaluated groups of patients was the expansive gas (SF6 or C3F8). The use of silicon oil was higher in 
Table 1 Baseline characteristics of patients in both subgroups

\begin{tabular}{|c|c|c|c|c|}
\hline Parameter & $\begin{array}{l}\text { All Patients } \\
(N=291)\end{array}$ & $\begin{array}{l}\text { Group A } \\
\text { High-myopic eyes } \\
\quad(N=67)\end{array}$ & $\begin{array}{l}\text { Group B } \\
\text { Non-high-myopic eyes } \\
\quad(N=224)\end{array}$ & $p$ value \\
\hline Age $($ mean $\pm \mathrm{SD})$ and range & $\begin{array}{l}60.2 \pm 12.5 \\
(18-86) \text { years }\end{array}$ & $\begin{array}{l}53.8 \pm 14.7 \\
(19-83) \text { years }\end{array}$ & $\begin{array}{l}62.1 \pm 11.1 \\
(18-86) \text { years }\end{array}$ & $<0.001$ \\
\hline Women $(\%)$ & $149(51.2 \%)$ & $40(59.7 \%)$ & $109(48.7 \%)$ & 0.113 \\
\hline $\begin{array}{l}\text { Follow-up time (mean } \pm \mathrm{SD} \text { ) and } \\
\text { range }\end{array}$ & $\begin{array}{l}30.6 \pm 22.8 \\
(6-96) \text { months }\end{array}$ & $\begin{array}{l}33.0 \pm 23.6 \\
(6-84) \text { months }\end{array}$ & $\begin{array}{l}29.9 \pm 22.6 \\
(6-96) \text { months }\end{array}$ & 0.333 \\
\hline Macula status & & & & 0.008 \\
\hline Macula on RD & $65(22.3 \%)$ & $7(10.4 \%)$ & $58(25.9 \%)$ & \\
\hline Macula-off RD & $226(77.7 \%)$ & $60(89.6 \%)$ & $166(74.1 \%)$ & \\
\hline Lens status & & & & 0.007 \\
\hline Phakic & $184(63.2 \%)$ & $40(59.7 \%)$ & $144(64.3 \%)$ & \\
\hline Pseudophakic & $98(33.7 \%)$ & $21(31.3 \%)$ & $77(34.4 \%)$ & \\
\hline Aphakic & $9(3.1 \%)$ & $6(9.0 \%)$ & $3(1.3 \%)$ & \\
\hline \multicolumn{5}{|l|}{ Duration of $R D$ prior to surgery } \\
\hline In days $<7$ days & $122(41.9 \%)$ & $25(37.3 \%)$ & $97(43.3 \%)$ & 0.383 \\
\hline$\geq 7$ days & $169(58.1 \%)$ & $42(62.7 \%)$ & $127(56.7 \%)$ & \\
\hline Type of surgery & & & & 0.167 \\
\hline $20 \mathrm{G}$ & $130(44.7 \%)$ & $25(37.3 \%)$ & $105(46.9 \%)$ & \\
\hline $23 \mathrm{G}$ & $161(55.3 \%)$ & $42(62.7 \%)$ & $119(53.1 \%)$ & \\
\hline Primary tamponade & & & & 0.135 \\
\hline Air & $4(1.4 \%)$ & - & $4(1.8 \%)$ & \\
\hline SF6 & $72(24.7 \%)$ & $11(16.4 \%)$ & $61(27.2 \%)$ & \\
\hline $\mathrm{C} 3 \mathrm{~F} 8$ & $170(58.4 \%)$ & $42(62.7 \%)$ & $128(57.1 \%)$ & \\
\hline SO & $45(15.5 \%)$ & $14(20.9 \%)$ & $31(13.8 \%)$ & \\
\hline Peripheral retinal degenerations & $47(16.2 \%)$ & $8(11.9 \%)$ & $39(17.4 \%)$ & 0.286 \\
\hline Number of retinal breaks and holes & & & & 0.869 \\
\hline 1 & $137(47.1 \%)$ & $33(49.3 \%)$ & $104(46.4 \%)$ & \\
\hline 2 & $73(25.1 \%)$ & $17(25.4 \%)$ & $56(25.0 \%)$ & \\
\hline 3 and more & $81(27.8 \%)$ & $17(25.4 \%)$ & $64(28.6 \%)$ & \\
\hline $\begin{array}{l}\text { Localization of retinal breaks and } \\
\text { holes }\end{array}$ & & & & 0.933 \\
\hline Superior & $174(59.8 \%)$ & $39(58.2 \%)$ & $135(60.3 \%)$ & \\
\hline Inferior & $48(16.5 \%)$ & $11(16.4 \%)$ & $37(16.5 \%)$ & \\
\hline Superior and inferior & $69(23.7 \%)$ & $17(25.4 \%)$ & $52(23.2 \%)$ & \\
\hline Number of quadrants of RRD & & & & 0.089 \\
\hline 1 & $25(8.6 \%)$ & $3(4.5 \%)$ & $22(9.8 \%)$ & \\
\hline 2 & $134(46.0 \%)$ & $25(37.3 \%)$ & $109(48.7 \%)$ & \\
\hline 3 & $78(26.8 \%)$ & $22(32.8 \%)$ & $56(25.0 \%)$ & \\
\hline 4 & $54(18.6 \%)$ & $17(25.4 \%)$ & $37(16.5 \%)$ & \\
\hline
\end{tabular}

eyes with high myopia (group A) but did not differ significantly from group B [14 of $63(21 \%)$ vs. 31 of 224 (14\%) eyes; $p=0.178]$.
Functional results

The baseline BCVA was significantly lower in eyes with high myopia (1.58 LogMAR) when compared 
Fig. 1 The anatomical success rates after the primary surgery and after the following surgeries at evaluated time points

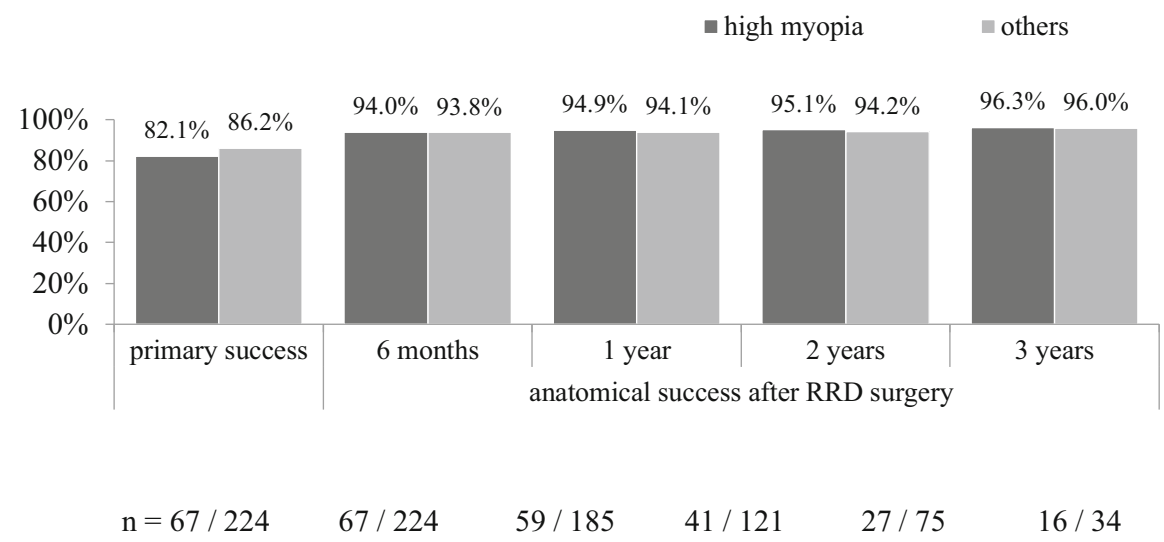

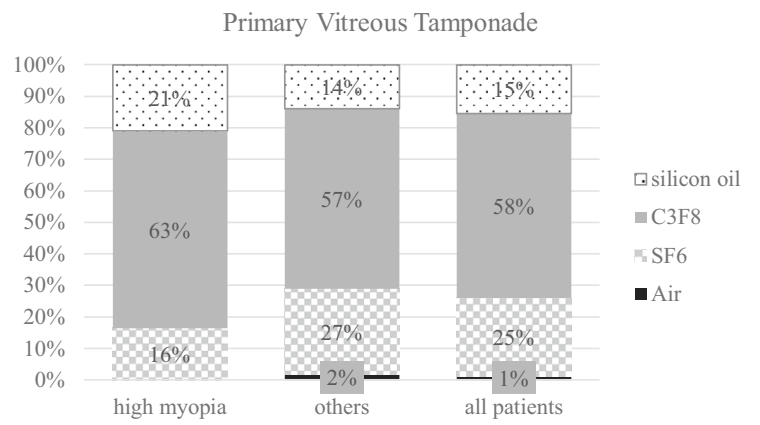

Fig. 2 The used vitreous tamponade at the end of the primary surgery in all eyes and in both subgroups separately

with 1.21 LogMAR in non-high-myopic eyes $(p<0.001)$. Figure 3 shows the development of BCVA in both subgroups during the follow-up of 3 years. The differences between group A and group B at month 6; years 1,2 and 3 were statistically significant $(p<0.001 ; p<0.001, p=0.011 ;$ and $p=0.025$, respectively).
At baseline, 10.4\% high-myopic eyes and 20.5\% non-high-myopic eyes presented the BCVA $\leq 0.3$ LogMAR $(p=0.071)$. The prevalence of BCVA $\leq 0.3$ LogMAR remained significantly higher in high-myopic eyes during the entire analysed followup of 3 years (see Fig. 4).

\section{Central retinal thickness}

The mean central retinal thickness (CRT) at month 6 after initial RD surgery was in group A $233 \pm 42 \mu \mathrm{m}$ and in group B $264 \pm 75 \mu \mathrm{m}$. No significant changes in CRT were observed within each single subgroup during the follow-up period of 3 years (see Fig. 2). The CRT values at each follow-up time point are presented in Fig. 5. At each evaluated visit, significantly lower CRT value was documented in highmyopic eyes when compared with non-high-myopic eyes.
Fig. 3 Changes in BCVA (LogMAR) after the primary surgery in both study subgroups

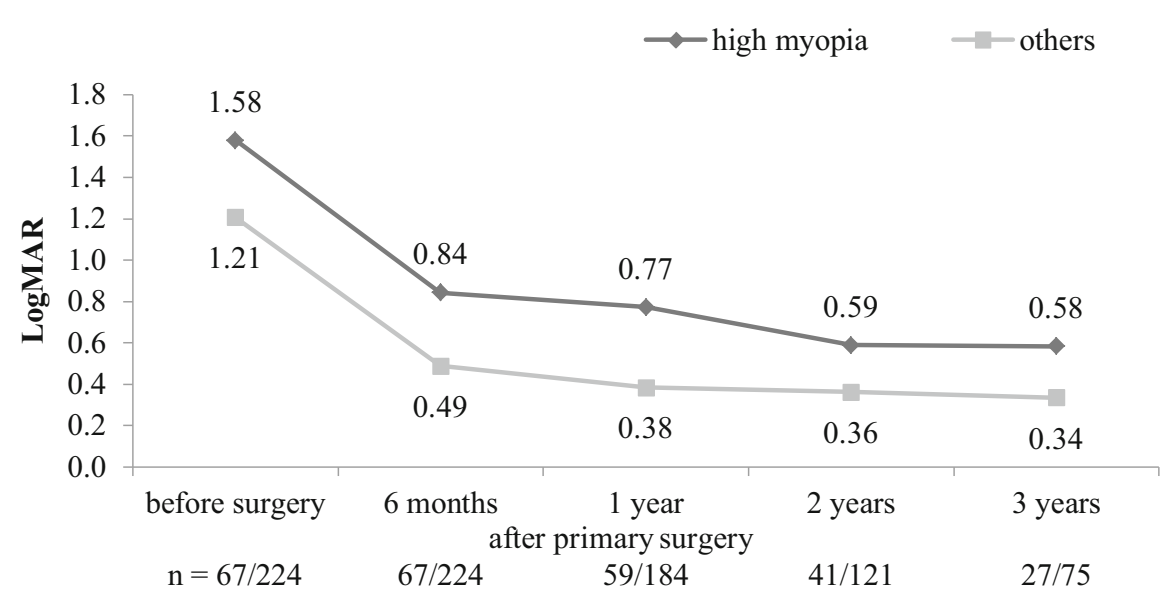


Fig. 4 The prevalence of BCVA $\leq 0.3$ LogMAR in both study subgroups during the entire follow-up time of three years

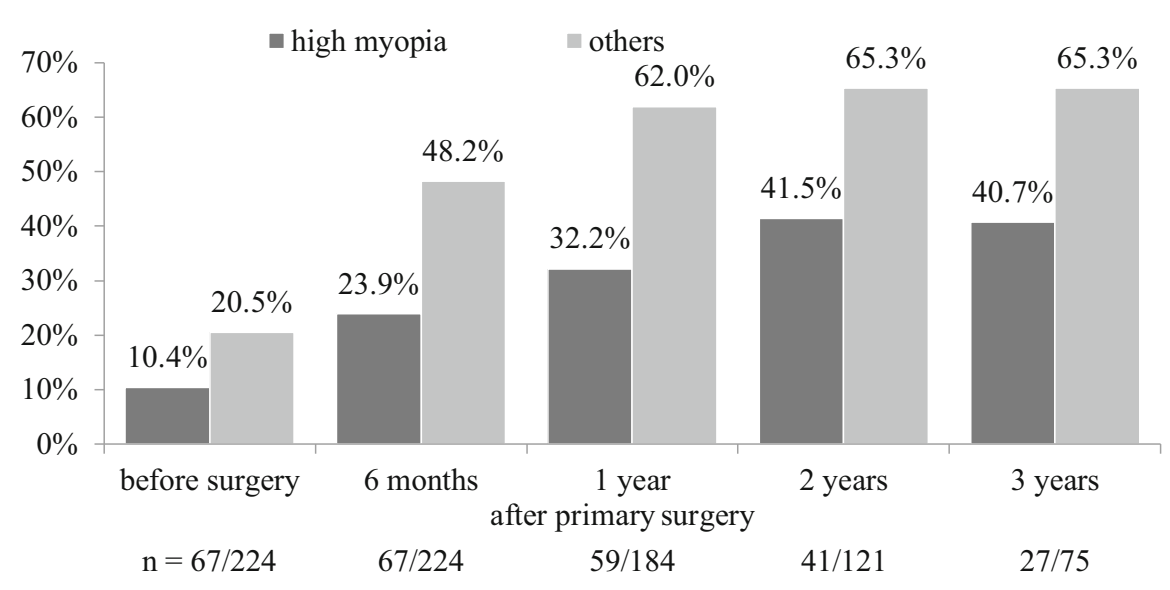

Fig. 5 CRT after the primary surgery

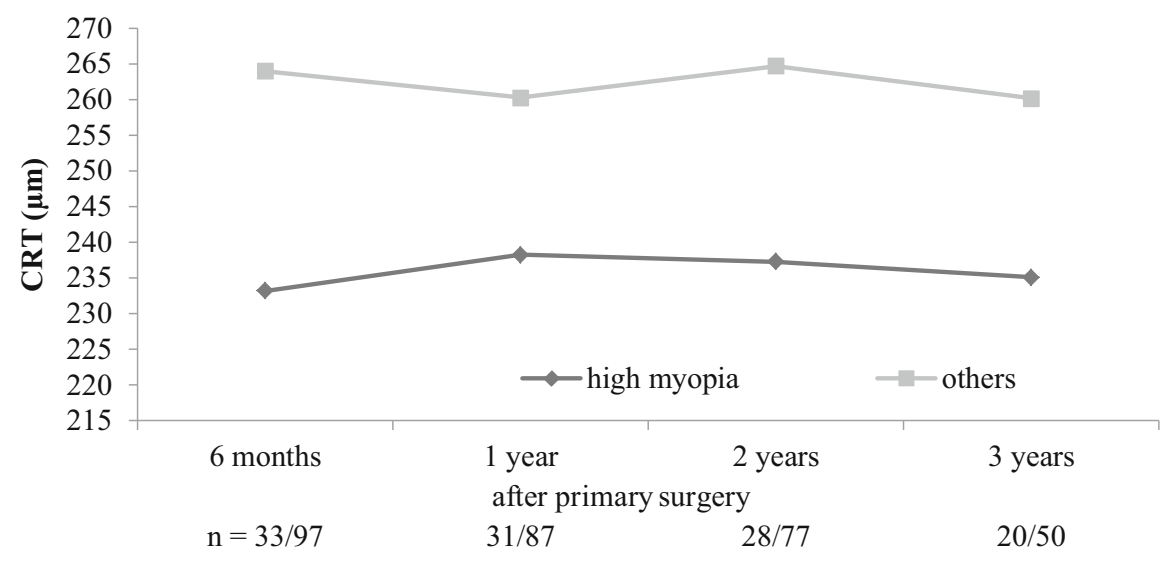

As the prevalence of macula-off RRD differed significantly between eyes with (group A) and without (group B) high myopia and even non-significant, but obvious, difference in the use of silicon oil tamponade was presented, we performed an additional matchedpair analysis of paired eyes from both subgroups to evaluate the changes in BCVA and CRT. Reflecting the fact that in group A most eyes presented with macula-off RRD, we used for this subanalysis the following eyes: (1) macula-off RRD, (2) absence of Aphakia, (3) PPV without primary ILM-peeling (3) primary tamponade with gas (SF6 or $\mathrm{C} 3 \mathrm{~F} 8$ ) and (4) the absence or other pathologies affecting the BCVA and CRT (e.g. amblyopia, macular hole, etc.)

The matched pairs were built from all in the database-identified eligible eyes. For each eye with high myopia in group A (in total 67 patients), a matched patient from group B (from total 224 patients) was identified by matching for gender, age (with difference $\leq 5$ years) and BCVA (with difference $\leq 0.1$ LogMAR). Cases for whom a matched pair could not be appropriately identified were dropped from the subsequent analysis. Whenever multiple matches were possible, the non-myopic eyes with the closest BCVA value to the myopic eye were selected. Data were manually cross-checked after export to SPSS, with random-sampling verification of the matching process.

In total, 20 matched pairs could be created. The changes in BCVA and CRT during the follow-up time over 3 years are shown in Figs. 6 and 7, respectively. Even the non-high-myopic eyes presented better BCVA after the primary surgery, and the CRT in high-myopic eyes tends to decrease stronger than in group B; in both evaluated functional parameters, no significant differences between high-myopic and nonhigh-myopic eyes could be detected during the entire follow-up time. 
Fig. 6 Changes in BCVA in matched pairs of eyes with primary macula-off RRD treated with primary PPV with expansive gas

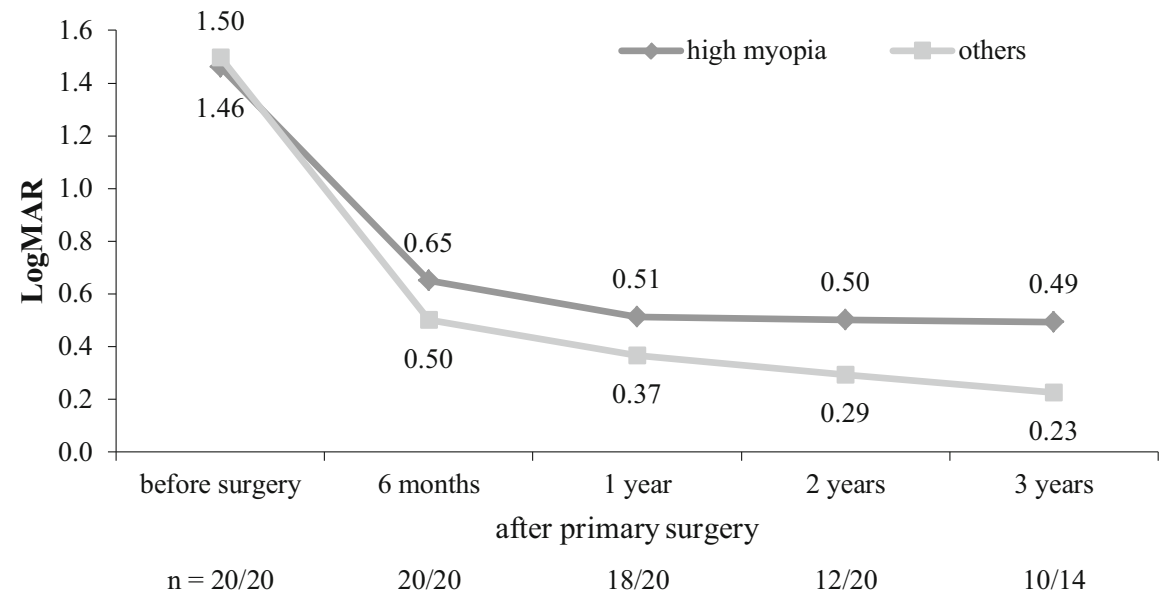

Fig. 7 Changes in CRT in matched pairs of eyes with primary macula-off RRD treated with primary PPV with expansive gas

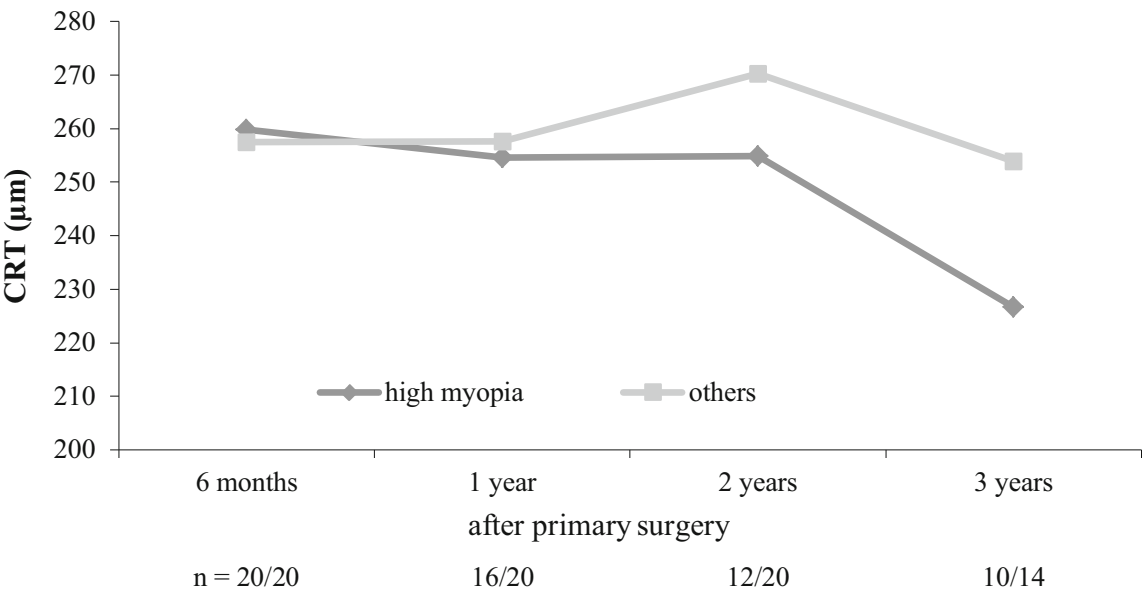

\section{Discussion}

In our study, the SSSR in eyes with high myopia was $82.1 \%$ and the final reattachment rate after 3 years was $96.3 \%$. The results did not significantly differ from anatomical results in non-high-myopic eyes (SSSR 86.2\%; final reattachment rate $96.0 \%, p>0.05$ ). Our results are in line with other published studies. Kwok et al. [8] reported the primary anatomical success rate in the amount of $86.1 \%$ in the scleral buckle (SB) group and $75 \%$ in the PPV group concerning the high-myopic eyes. The final reattachment rate was $97.2 \%$ for the SB group and $100 \%$ for the PPV group. Cheng et al. [9] reported the anatomical success rate in the amount of $86.5 \%$ after the primary vitreoretinal surgery and the final anatomical success rate was $100 \%$. Bernheim et al. [10] showed in their prospective study the final reattachment rate referring to the high myopia in the amount of $96 \%$. The increased use of PPV, already described in association with the other diseases, reduces the operating time, allows better visualization of breaks, increases the single retinal reattachment rate $[8,9]$ and reduces the refractive changes compared to the scleral buckle surgery [11-14]. Due to the anatomical specifics, the RDD surgery of high-myopic eyes is challenging and the SSSR was reported to be lower than that in nonmyopic eyes [6]. The need for adjuvant buckle (encircling band) combined with the primary PPV in highmyopic eyes with RDD is still discussed and not finally clarified yet. The results of VIPER Study are reporting success rates of the primary PPV versus PPV combined with encircling band (EB) in pseudophakic eyes [15]. No significant difference was observed among groups treated with 20G PPV with adjuvant EB (79.0\%) when compared with eyes treated with 20G without EB (78.7\%) and 23/25G without EB (87.7\%) [15]. 
Ripandelli et al. [16] described the benefit form additional buckle with inferior indentation in recurrent retinal detachment in highly myopic eyes. Based on the results of our analysis, there is no need for primary adjuvant buckle (EB) in all myopic eyes.

The postoperative functional outcomes in highmyopic eyes are less satisfactory than the anatomical results. In our study the high-myopic eyes reached 3-years after primary surgery the high-myopic eyes reached the BCVA of 0.58 LogMAR compared to 0.34 LogMAR in non-high-myopic eyes. At this time, point BCVA of $\leq 0.3$ was achieved in $40.7 \%$ of highmyopic eyes.

Non-high-myopic eyes presented significantly more favourable visual prognosis. This observation correlates with significantly worse baseline (before RRD surgery) BCVA in high-myopic eyes. Further, the worse functional results might be caused also by the observed CRT decrease in this subgroup. It is also important to consider that 60 of 67 myopic eyes (89.6\%) presented the macula-off detachment. In group B (non-high-myopic eyes), the macula-off detachment was preoperatively presented in 166 of 224 eyes $(74.1 \%$ ) only. The BCVA of $\leq 0.3$ LogMAR reached $65.3 \%$ of patients in this subgroup. Kwok et al. [8] reported that $62.5 \%$ of the eyes undergoing the primary pars plana vitrectomy reached the visual acuity of 20/60 or better. Cheng et al. [9] demonstrated that $26.3 \%$ of eyes achieved the postoperative vision $20 / 50$ or better in the pars plana group and $68.5 \%$ of the eyes in the scleral buckling group. The obvious difference in functional result between both groups could be explained by the fact that the vitrectomy was used only in more complicated RD cases. Bernheim et al. [10] reported that $54 \%$ of the eyes after the first surgery and $44 \%$ of the eyes after the multiple surgeries had the visual acuity $20 / 40$ or better. The good visual prognosis was negatively associated with the presence and duration of macular detachment and the axial length. Rodriguez et al. [17] in his study reported that $65 \%$ of the eyes with high myopia had the final visual acuity $20 / 40$ or better after the 6-month follow-up concerning the scleral buckling surgery. Visual recovery after RD surgery is still unclear, and it does not depend on the entity of the RD. Vignolo et al. [18] demonstrate that in biofeedback group, there was a significant recovery in visual performances that still remains evident after 3 months from the baseline.
Further, our study confirmed the knowledge that CRT in high-myopic eyes is significantly lower than that in non-high-myopic eyes. However, after PPV for RD no significant differences in development of CRT were observed between both groups. The analysis of all high-myopic versus non-high-myopic eyes showed the trend to decrease of CRT in high-myopic eyes. However, in the matched-paired analysis, in which the eyes with macula-off RDD myopic eyes presented with lower CRT, no significant decrease during the follow-up of 3 years after the primary surgery was observed when compared with non-high-myopic eyes.

Our study is a retrospective analysis, underlying all limitations of a non-randomized trial. The largest limitation is the small sample size. Furthermore, it could not be excluded that some patients who underwent the primary surgery in our clinic and had further need for repetitive surgeries did not missed their follow-up visit and underwent their surgery elsewhere. Further limitation of the study is the exclusion of eyes with PVR grade C.

In conclusion, the results of our study showed that the anatomical success rate of primary PPV without adjuvant buckle in high-myopic eyes is comparable with the results in eyes without high myopia. However, in the subgroup with high myopia we obtained a significant decrease in the BCVA and CRT on OCT. Similar anatomical results of the pars plana vitrectomy without adjuvant procedures were referred by other authors in eyes without myopia [19-22].

Significant advances of the surgical treatment of RRD have been made, and a variety of techniques are now available, with new instruments and modifications constantly being reported. However, further improvements are expected in the future [23, 24].

\section{Compliance with ethical standards}

Conflict of interest Author Ladislav Janco declares that he has no conflict of interest. Author Katarina Tkacova-Villemova declares that she has no conflict of interest. Author Maria Mesarosova declares that she has no conflict of interest. Author Marta Ondrejkova declares that she has no conflict of interest. Author Alexandra Kollarova declares that she has no conflict of interest. Author Matus Rehak declares that he has no conflict of interest.

Ethical approval All procedures performed in studies involving human participants were in accordance with the ethical standards of the institutional and/or national research committee and with the 1964 Helsinki Declaration and its later amendments or comparable ethical standards. 
Informed consent Informed consent was obtained from all individual participants included in the study.

Open Access This article is distributed under the terms of the Creative Commons Attribution 4.0 International License (http:// creativecommons.org/licenses/by/4.0/), which permits unrestricted use, distribution, and reproduction in any medium, provided you give appropriate credit to the original author(s) and the source, provide a link to the Creative Commons license, and indicate if changes were made.

\section{References}

1. Holden BA, Fricke TR, Wilson DA, Jong M, Naidoo KS, Sankaridurg P, Wong TY, Naduvilath TJ, Resnikoff S (2016) Global prevalence of myopia and high myopia and temporal trends from. 2000 through 2050. Ophtalmology 123:1036-1042

2. Hopf S, Pfeiffer N (2017) Epidemiology of myopia. Ophthalmologe 114:20-23

3. Morgan IG, He M, Rose KA (2017) Epidemic of pathologic myopia: what can laboratory studies and epidemiology tell us? Retina 37:989-997

4. Kung YH, Wu TT (2016) Risk factors of hyphema following surgeries for primary rhegmatogenous retinal detachment. Ophthalmologica 236:159-165

5. Smretschnig E, Falkner-Radler CI, Spörl J, Kivaranovic D, Binder S, Krepler K (2017) Primary retinal detachment surgery: changes in treatment and outcome in an Austrian Tertiary Eye Center. Ophtalmologica 237:55-62

6. Javorska L, Krasnik V, Vavrova K, Strmeň P (2015) The changes of the spectrum in primarily indicated surgeries due to retinal detachment during the period of 15 years. Cesk Slov Oftalmol 71:93-99

7. Bach M, Kommerell G (1998) Determining visual acuity using European normal values: scientific principles and possibilities for automatic measurement. Klin Monbl Augenheilkd 212:190-195

8. Kwok KA, Cheng LL, Tse MW, Cheung EY, Lam DS (2002) Outcomes of primary rhegmatogenous retinal detachment in myopes of five or more diopters. Ophtalmic Surg Lasers 33:188-194

9. Cheng SF, Yang CH, Lee CH, Yang CM, Huang JS, Ho TC, Lin CP, Chen MS (2008) Anatomical and functional outcome of surgery of primary rhegmatogenous retinal detachment in high myopic eyes. Eye 22:70-76

10. Bernheim D, Rouberol F, Palombi K, Albrieux M, Romanet JP, Chiquet CH (2013) Comparative prospective study of rhegmatogenous retinal detachments in phakic or pseudophakic patients with high myopia. Retina 33:2039-2048

11. Brazitikos PD, Androudi S, Christen WG, Stangos NT (2005) Primary pars plana vitrectomy versus scleral buckle surgery for the treatment of pseudophakic retinal detachment: a randomized clinical trial. Retina 25:957-964

12. Heimann H, Bartz-Scmidt KU, Bornfeld N et al (2007) Scleral buckling versus primary vitrectomy in rhegmatogenous retinal detachment: a prospective randomized multicenter clinical study. Ophtalmology 114:2142-2154
13. Falkner-Radler CI, Myung JS, Moussa S, Paul Chan RV, Smretschnig E, Kiss S, Graf A, D'amico DJ, Binder S (2011) Trends in primary retinal detachment surgery: results of a Bicenter Study. Retina 31:928-936

14. Sun Q, Sun T, Xu Y, Yang XL, Xu X, Wang BS, Nishimura T, Heimann H (2012) Primary vitrectomy versus sclera buckling for the treatment of rhegmatogenous retinal detachment: a meta- analysis of randomized controlled clinical trials. Curr Eye Res 37:492-499

15. Walter P, Hellmich M, Baumgarten S, Schiller P, Limburg E, Agostini H, Pielen A, Helbig H, Lommatzsch A, Rössler G, Mazinani B (2017) VIPER Study Group. Vitrectomy with and without encircling band for pseudophakic retinal detachment: VIPER Study Report no 2-main results. Br J Ophthalmol 101:712-718

16. Ripandelli G, Rossi T, Scarinci F, Stirpe M (2015) Encircling scleral buckling with inferior indentation for recurrent retinal detachment in highly myopic eyes. Retina 35:416-422

17. Rodriguez FJ, Lewis H, Kreiger AE et al (1991) Scleral buckling for rhegmatogenous retinal detachment associated with severe myopia. Am J Ophtalmol 111:595-600

18. Vignolo EM, Fragiotta S, Domanico D, Limoli Nebbioso M, Spadea L (2016) Visual recovery after primary retinal detachment surgery: biofeedback rehabilitative strategy. J Ophtalmol 2016:8092396. https://doi.org/10.1155/2016/ 8092396

19. Figueroa MS, Contreras I, Noval S (2013) PACORES Study Group: anatomic and visual outcomes of $23-\mathrm{G}$ vitrectomy without scleral buckling for primary rhegmatogenous retinal detachment. Eur J Ophtalmol 23:412-422

20. Schneider EW, Geraets RL, Johnson MW (2012) Pars plana vitrectomy without adjuvant procedures for repair of primary rhegmatogenous retinal detachment. Retina 32:213-219

21. Brazitikos PD (2000) The expanding role of primary pars plana vitrectomy in the treatment of rhegmatogenous noncomplicated retinal detachment. Semin Ophthalmol 15:65-67

22. Wong CW, Wong WL, Yeo IY, Loh BK, Wong EY, Wong DW, Ong SG, Ang CL, Lee SY (2014) Trends and factor related to outcomes for primary rhegmatogenous retinal detachment surgery in a large Asian tertiary center. Retina 34:684-692

23. Nemet A, Moshiri A, Yiu G, Loewenstein A, Moisseiev E (2017) A review of innovations in rhegmatogenous retinal detachment surgical techniques. J Ophthalmol 2017:4310643. https://doi.org/10.1155/2017/4310643

24. Rizzo S, Polizzi S, Barca F, Caporossi T, Virgili G (2017) Comparative study of 27-gauge versus 25-gauge vitrectomy for the treatment of primary rhegmatogenous retinal detachment. J Ophthalmol 2017:6384985. https://doi.org/ $10.1155 / 2017 / 6384985$

Publisher's Note Springer Nature remains neutral with regard to jurisdictional claims in published maps and institutional affiliations. 